\title{
Experimental glucocorticoid-induced bone loss in mice is strongly influenced by the strain
}

\author{
Adel Ersek ${ }^{1}$, Youridies Vattakuzhi ${ }^{2}$, Andrew R. Clark ${ }^{2}$ and Nicole J. Horwood ${ }^{1}$
}

1 The Kennedy Institute of Rheumatology, University of Oxford, UK, ${ }^{2}$ Imperial College London, London, UK.

\section{INTRODUCTION}

Glucocorticoids (GC) are often used as anti-inflammatory agents in the treatment of inflammatory disorders. However, long term use of GC in all chronic inflammatory diseases may produce unwanted secondary side effects such as GC induced osteoporosis (GIO). Experimental models of GIO are well established in the Swiss and CD1 mice, nonetheless most genetically modified mice are on C57BL/6 background.

The aim of this study was to investigate the effect of long term GC administration on bone turnover in two frequently used mouse strains; C57BL/6 and CD1 in order to assess the influence of genetic background on GIO in these strains.

\section{METHODS}

Bone marrow (BM) cells were used to investigate the effect of GCs on osteoclast (OC) in vitro activity.

GIO was induced in 12 weeks old female C57BL/6 and CD1 mice by subcutaneous insertion of long term (60 days) release prednisolone $3.2 \mathrm{mg} / \mathrm{kg} /$ day or placebo pellets, and bone architecture and biomechanical properties were evaluated.

RESULTS: The GC Dexamethasone promotes osteoclastogenesis in the CD1 mice BM in vitro.

By using BM cells that were induced to form OC, we have found that osteoclastogenesis was stimulated in a dose dependent manner by $C G$ in the $\mathrm{CD} 1$ mice BM but not in the $\mathrm{C} 57 \mathrm{BL} / 6$ mice BM.

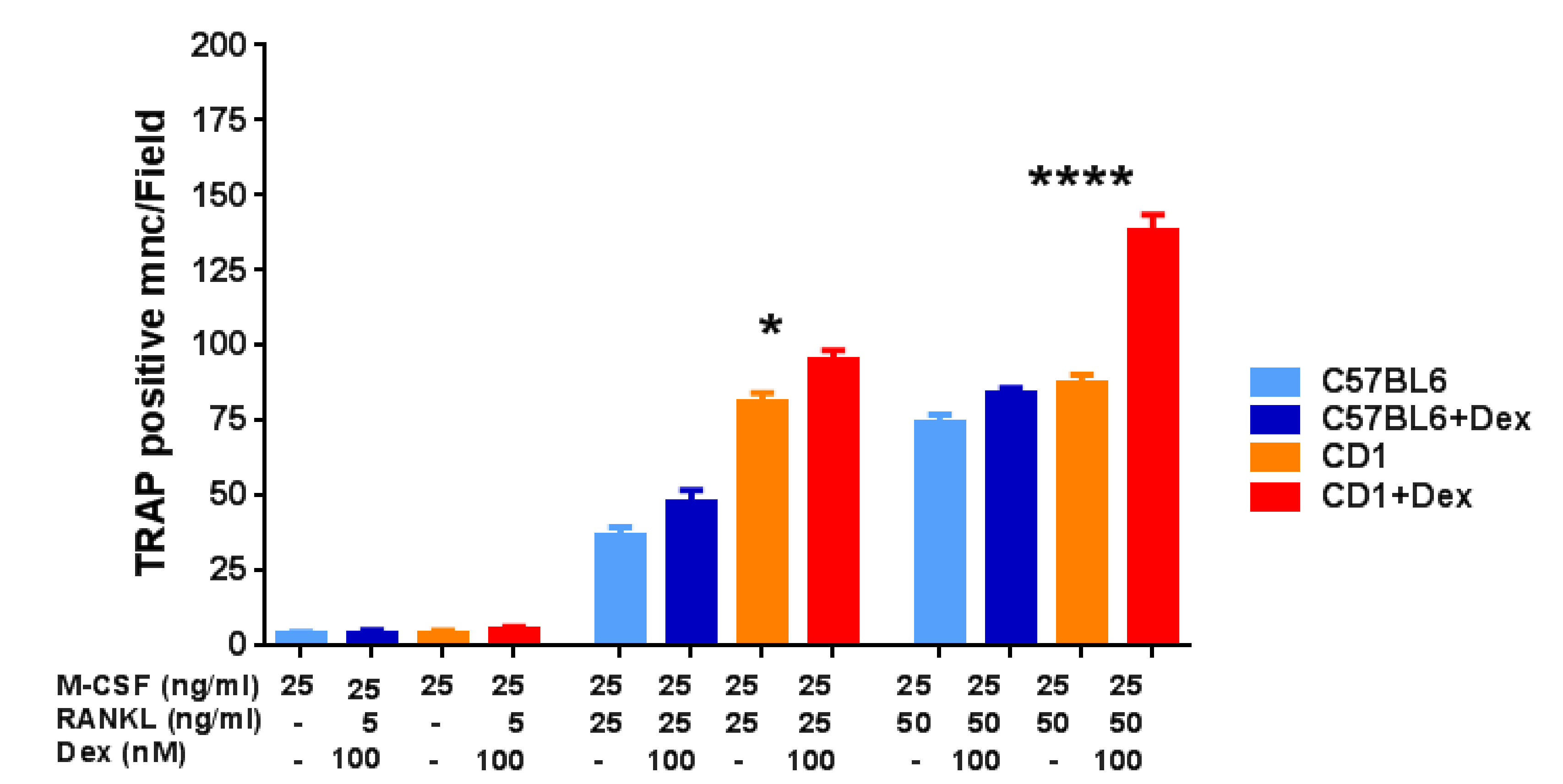

Figure 1: Enumeration of multinucleated $O C$ after tartrate resistant acid phosphatase (TRAP) staining. Cells were cultured in the presence of $25 \mathrm{ng} / \mathrm{ml} \mathrm{M-CSF}$ and $5-50 \mathrm{ng} / \mathrm{ml}$ RANKL for 7 days $\left({ }^{*} \mathrm{p}<0.05,{ }^{* * *} \mathrm{p}<0.001\right)$

RESULTS: Biomechanical properties of femur are reduced in GC treated CD1 mice but not in C57BL/6 mice.

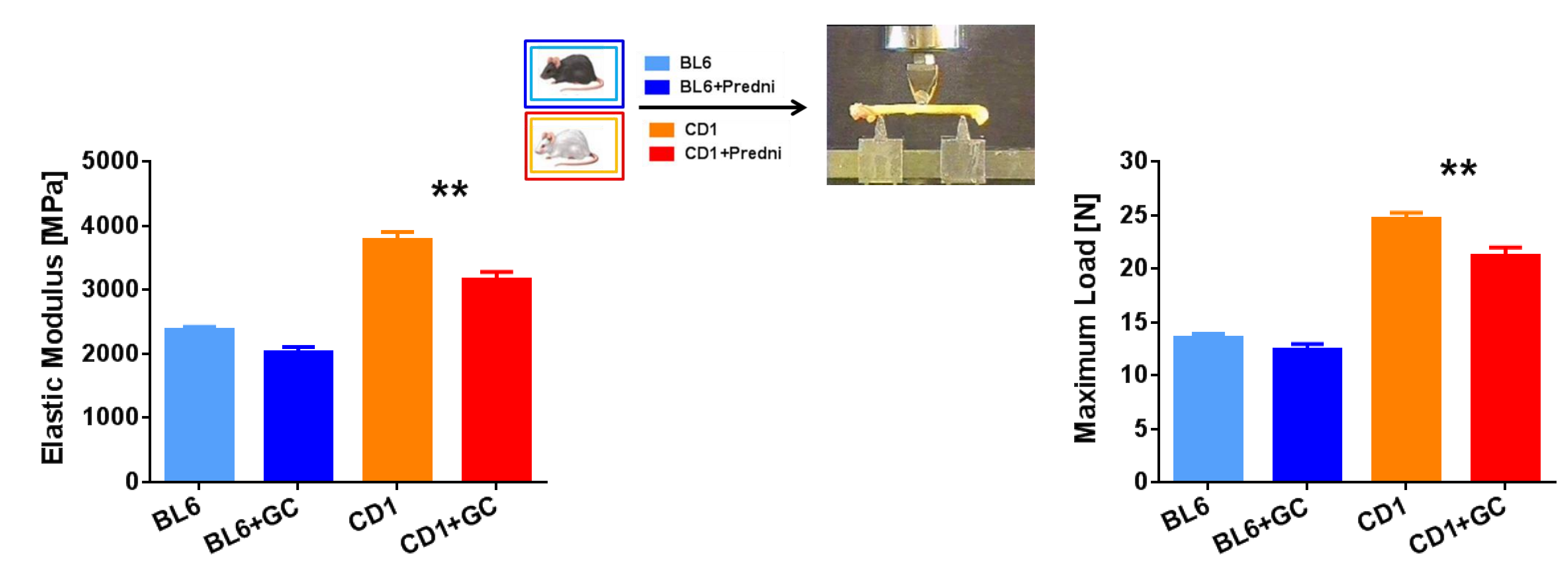

Figure 2: Biomechanical properties of femur evaluated by the three-point bending testing. Three-point bend testing was performed with Instron 5942 system. Based on the load-deformation curve, the maximum load and elastic modulus was determined by using Bluehill 3 software.

RESULTS: Long term in vivo Prednisolone administration induced osteoporosis in CD1 mice but not in C57BL/6 mice.

Micro-CT analysis of lumbar vertebrae revealed that long term GC administration reduced trabecular bone volume and trabecular number, while the structural model index was increased in CD1 mice as reported previously in GIO.

Bone morphometric parameters did not change in $\mathrm{GC}$ receiving or control C57BL/6 mice.
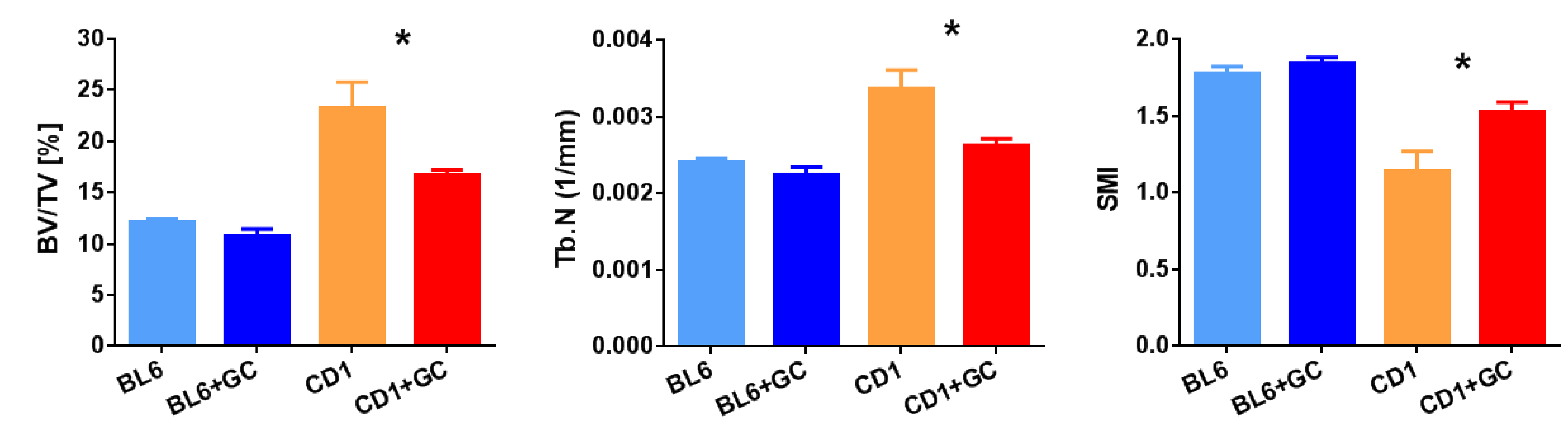

Figure 3: Bone indices of Prednisolone or Placebo treated mice obtained after micro-CT reconstruction. Lumbar vertebrae were scanned with Skyscan 1174 scanner and reconstructed images were analysed using Skyscan CT Analyzer software version 1.13.2.1 $\left({ }^{*} \mathrm{p}<0.05\right)$.

RESULTS: Long term GC treatment promotes OC activity in the CD1 mice in vivo but not in $\mathrm{C} 57 \mathrm{BL} / 6$ mice.

The number of osteoclasts per total bone area (N.Oc/T.Ar) and OC surface expressed as percent of total bone surface (Oc.S/BS) were significantly increased in the Prednisolone treated CD1 mice but not in C57BL/6 mice.
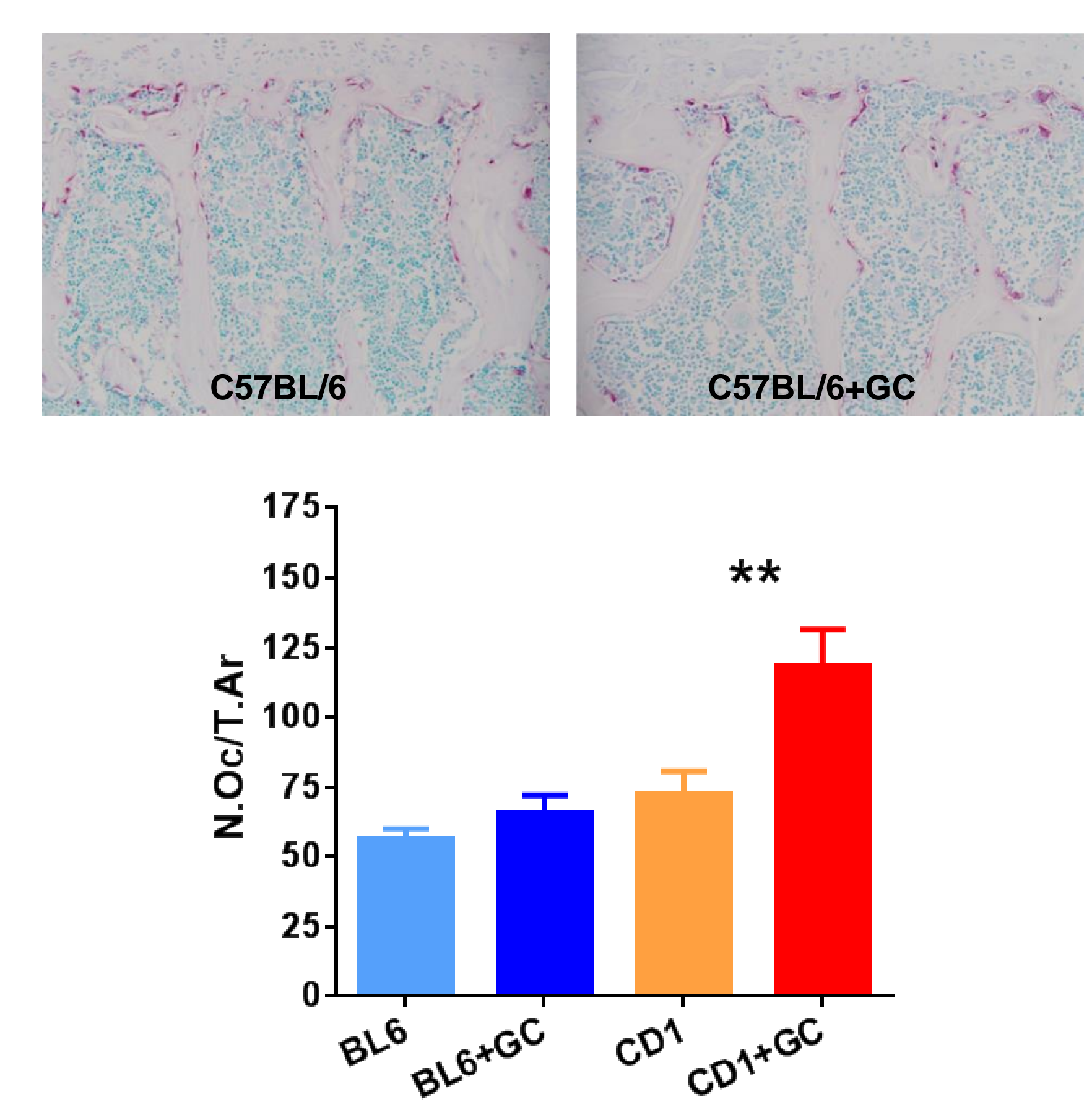

Figure 4: Histological sections of mouse lumbar vertebrae stained for OC TRAP activity. Lumbar vertebrae stained for OC TRAP activity and counterstained with $0.25 \%$ methyl green solution (magnification 20x) Bone histomorphometry parameters were obtained by analysing the histological sections $\left({ }^{* *} \mathrm{p}<0.01\right)$

\section{CONCLUSIONS}

Dexamethasone increased significantly the osteoclastogenic potential of CD1 mice BM in vitro while the C57BL6 mice BM was not affected.

Long term GC administration in vivo reduced maximum load and femoral elasticity, vertebral bone volume and trabecular number in CD1 mice while the biomechanical properties and bone indices were not affected in C57BL/6 mice.

Prednisolone stimulated in vivo osteoclastogenesis in the CD1 mouse but not in C57BL/6 mice.

Long term GC administration produced strain dependent differences; inducing GIO in CD1 mice while the C57BL6 mice appeared to be protected against $\mathrm{GIO}$ 